\title{
Syphilis se manifestant par une pelade
}

\author{
Meng Yin Wu MD, Jun Li MD
}

— Citation : CMAJ 2021 January 25;193:E126. doi : 10.1503/cmaj.200894-f

Voir la version anglaise de l'article ici : www.cmaj.ca/lookup/doi/10.1503/cmaj.200894

$\mathbf{U}$ $\mathrm{n}$ homme de 38 ans est venu consulter en dermatologie pour une perte de cheveux progressive depuis 6 mois. Il ne ressentait aucune démangeaison $\mathrm{ni}$ douleur au cuir chevelu, qui était parsemé de lésions semblables à des plaques chauves diffuses non cicatricielles et non inflammatoires, aux bords estompés et de taille irrégulière (figure 1A). Trois mois plus tôt, le patient avait présenté un érythème non prurigineux au tronc et aux membres, qui s'est résorbé spontanément en 10 semaines. Il a déclaré avoir eu des relations sexuelles non protégées 6 mois plus tôt et a dit ne pas avoir consommé de drogues injectables.

La recherche sérologique de la syphilis a donné des résultats positifs au test rapide de la réagine plasmatique (test RPR) (1:64; valeur normale [test négatif] $\leq 1: 8$ ) et au test d'immunofluorescence absorbée, appuyant ainsi le diagnostic de syphilis secondaire. Le dépistage du VIH a donné des résultats négatifs. Nous avons alors prescrit un traitement à la pénicilline par voie intramusculaire (3 doses de 2,4 millions UI par semaine). Trois mois plus tard, les cheveux du patient avaient repoussé (figure 1B), et le résultat au test RPR était de 1:8. Au suivi à 1 an, ce résultat était de 1:1.

$L^{\prime}$ 'incidence de la syphilis est en hausse au Canada ${ }^{1,2}$. Une pelade sans cicatrices ou démangeaisons a été observée chez environ 3\%-7\% des patients atteints de syphilis secondaire ${ }^{3}$. L'alopécie syphilitique - qui peut être en plaques, diffuse ou les deux à la fois - touche généralement les régions occipitale et pariétale. La perte de cheveux peut être accompagnée d'un érythème généralisé non prurigineux ou d'une lymphadénopathie généralisée non douloureuse, ou encore être précédée d'un chancre génital ${ }^{4}$. Chez les patients qui obtiennent un résultat positif au dépistage sérologique de la syphilis, il faut envisager un diagnostic d'alopécie essentielle si l'alopécie est la seule manifestation de l'infection, et un diagnostic d'alo- pécie symptomatique si la perte de cheveux est accompagnée d'autres signes et symptômes de syphilis ${ }^{3}$.

À la trichoscopie, les signes cliniques évocateurs d'autres diagnostics comprennent la présence de cheveux « en point d'exclamation », laissant croire à une alopécie en aires, et celle de cheveux « en virgule », pouvant caractériser la teigne du cuir chevelu ${ }^{5}$. Les antécédents d'arrachage de cheveux et l'observation de cheveux cassés de façon irrégulière à la trichoscopie sont des indices de trichotillomanie.

\section{Références}

1. Choudhri Y, Miller J, Sandhu J, et al. Infectious and congenital syphilis in Canada, 2010-2015. Can Commun Dis Rep 2018;44:43-8.

2. Infectious syphilis cases reported in Canada 2009-2018 [infographique]. Can Commun Dis Rep 2019;45-11:302. Accessible ici : www.canada.ca/en/public -health/services/reports-publications/canada-communicable-disease-report-ccdr /monthly-issue/2019-45/issue-11-november-7-2019/article-5-infectious-syphilis -canada-2009-2018.html (consulté le 18 juin 2020). 
3. Piraccini BM, Broccoli A, Starace M, et al. Hair and scalp manifestations in secondary syphilis: epidemiology, clinical features and trichoscopy. Dermatology 2015;231:171-6

4. Li S, Wu L. Hair loss and lymphadenopathy. BMJ 2019;366:14555.

5. Rudnicka L, Rakowska A, Kerzeja M, et al. Hair shafts in trichoscopy: clues for diagnosis of hair and scalp diseases. Dermatol Clin 2013;31:695-708.

Intérêts concurrents : Aucun déclaré.

Cet article a été révisé par des pairs.

Les auteurs ont obtenu le consentement du patient.

Affiliation : Département de dermatologie, Hôpital médical universitaire Union de Pékin, Académie chinoise de sciences médicales et Collège médical Union de Pékin, Beijing, Chine.

Propriété intellectuelle du contenu : Il s'agit d'un article en libre accès distribué conformément aux modalités de la licence Creative Commons Attribution (CC BY-NC-ND 4.0), qui permet l'utilisation, la diffusion et la reproduction dans tout médium à la condition que la publication originale soit adéquatement citée, que l'utilisation se fasse à des fins non commerciales (c.-à-d., recherche ou éducation) et qu'aucune modification ni adaptation n'y soit apportée. Voir : https://creativecommons.org/licenses/by-nc-nd/4.0/deed.fr.

Correspondance : Jun Li, lijun35@hotmail.com

Les images cliniques sont choisies pour leur caractère particulièrement intéressant, classique ou impressionnant. Toute soumission d'image de haute résolution claire et bien identifiée doit être accompagnée d'une légende aux fins de publication. On demande aussi une brève explication (300 mots maximum) de la portée éducative des images, et des références minimales. Le consentement écrit du patient au regard de la publication doit être obtenu avant la soumission. 